\title{
The depressor caudal ventrolateral medulla: Its correlation with the pressor dorsomedial and ventrolateral medulla and the depressor paramedian reticular nucleus
}

\author{
J.H. Hsieh, J.J. Wu, C.T. Yen, C.Y. Chai * \\ Institute of Biomedical Sciences, Academia Sinica, Institute of Biomedical Engineering, Chung Yuan Christian University, Department of Zoology, \\ National Taiwan University, Taipei, Taiwan
}

Received 8 July 1997; revised 6 February 1998; accepted 6 February 1998

\begin{abstract}
The functional correlation of the depressor caudal ventrolateral medulla (CVLM) with the two pressor regions, i.e. rostral ventrolateral medulla (RVLM) and dorsomedial medulla (DM), and with another inhibitory region, i.e. the paramedian reticular nucleus (PRN), were studied in cats anesthetized intraperitoneally with chloralose $(40 \mathrm{mg} / \mathrm{kg})$ and urethane $(400 \mathrm{mg} / \mathrm{kg})$. Systemic arterial pressure (SAP), heart rate (HR) and the sympathetic vertebral nerve activity (VNA) were recorded. The correct location of CVLM, RVLM or DM was determined by their sepecific responses, i.e. decreases of SAP, HR and VNA, for CVLM increases of these parameters for RVLM and DM, elicited first by electrical stimulation $(80 \mathrm{~Hz}, 0.5 \mathrm{~ms}, 50-100 \mu \mathrm{A})$ then followed by microinjection of glutamate (Glu, $0.25 \mathrm{M}, 70$ nl). The depressor action of PRN was produced by electrical stimulation only. It was found that the depressor responses caused by the CVLM stimulation were greatly reduced $2 \mathrm{~h}$ after lesioning either the RVLM or DM by microinjection of kainic acid (KA, 24 mM, 200 nl) ipsilateral to the side of CVLM stimulation. The CVLM responses were further reduced after the remaining side of RVLM or DM was lesioned. The reduction of the CVLM-depressor responses was more apparent after the RVLM than DM lesioning. Data suggest that the CVLM-depressor responses are mediated through inhibition of the sympathetic-pressor neurons in both RVLM and DM with predominance of the former. Lesioning the PRN by KA and/or combination with DC electrolytic lesion reduced the CVLM-induced depressor responses. In turn, lesioning the CVLM by KA reduced the PRN-induced depressor responses. The reduction in the later manipulation was more apparent in the PRN-depressor responses than the CVLM-depressor responses. Data suggest that part of the PRN depressor action is mediated through activation of the CVLM. (C) 1998 Elsevier Science B.V. All rights reserved.
\end{abstract}

Keywords: Caudal ventrolateral medulla; Dorsomedial medulla; Rostral ventrolateral medulla; Sympathetic inhibition, Paramedian reticular nucleus

\section{Introduction}

The existence of neurons in the caudal ventrolateral medulla (CVLM) effecting vasodepressor action was first reported by Feldberg and Guertzenstein (1976) who found that in cats application of nicotine to the ventral surface of the medulla elicited a fall in blood pressure. This inspired a series of studies of various types leading to a final localization of the depressor action in the CVLM in the region between the nucleus ambiguous and the lateral reticular nucleus (Li and Blessing, 1990; Masuda et al.,

\footnotetext{
* Corresponding author. Institute of Biomedical Sciences Academia Sinica, Taipei 11529, Taiwan. Tel.: +886 2 27899105; fax: +8862 27829224.
}

1991; Willette et al., 1983). Neurons in the CVLM were first thought to be A1 adrenergic neurons. This was found not to be the case, as precise mapping of the depressor sites of CVLM in rats has shown that these sites are located outside the A1 cell group (Bonham and Jesek, 1989; Day et al., 1983). In fact, neurons in the CVLM, that respond to baroreceptor activation by i.v. phenylephrine and are labelled by injecting rhodamine latex bead into the rostral ventrolateral medulla (RVLM), are not immunoreactive to tyrosine hydroxylase, an enzyme essential for the synthesis of dopa (Badoer et al., 1994).

The depressor response evoked by CVLM activation is a result of decrease in the total peripheral resistance (Willette et al., 1987) consequent to a widespread inhibition of the sympathetic vasomotor activity. The effect is generally 
believed to be mediated through inhibition of the pressor neurons in the RVLM (Willette et al., 1984a,b). Indeed, by applying biocytin, an anterograde tracer, in the nucleus solitarius (NTS), and wheat germ agglutin-apo-horseradish peroxidase conjugated to colloid gold, a retrograde tracer, in the RVLM of the same animal at the same time, clear monosynaptic connections circuitry from the NTS to the CVLM, and also from the CVLM to the RVLM were evident (Aicher et al., 1995). Thus, the CVLM is not only important for mediating baroreceptor reflex but also for modulation of the medullary vasopressor action (Masuda et al., 1991). Neurons in the CVLM effecting sympathetic inhibition by baroreceptor activation reside in the rostral portion, while those causing sympathetic inhibition unrelated to baroreceptor reflex are distributed in the caudal portion (Cravo et al., 1991). The pathway is principally ipsilateral, because after division of the medulla at the midline, the reduction of spontaneous unit firing in the RVLM subsequent to glutamate (Glu) activation of the CVLM persisted (Masuda et al., 1991).

Pharmacological studies have shown that CVLM neurons receive both tonically active excitatory and inhibitory inputs. Blockade of $N$-methyl-D-aspartate (NMDA) receptors in the CVLM leads to a marked pressor response, indicating that Glu or other excitatory amino acids are tonically released there (Kubo and Kihara, 1988; Kubo et al., 1993). In addition, CVLM depressor neurons have been shown to be tonically inhibited by endogenous gamma-aminobutyric acid (GABA) and glycine (Blessing and Reis, 1983; Willette et al., 1984a,b). Other transmitters also have been found relating to CVLM activation. These include the angiotensin II (Sesoko et al., 1995), taurine and $\beta$-alanine (Kubo et al., 1993), opioid (Badoer and Chalmers, 1992) and neuropeptide Y (Macrae and Reid, 1988).

As was mentioned previously, it is generally accepted that CVLM effects depressor action through inhibition of the RVLM. In recent years, we (Chai et al., 1988, 1990, 1991, 1993a,b, 1997; Lin et al., 1989; Su et al., 1989), and others (Barman and Gebber, 1983; Goodchild and Dampney, 1985; Hirooka et al., 1994; Yardley et al., 1989) have repeatedly demonstrated the existence of neurons in the dorsomedial medulla (DM) responsive to excitatory amino acids in generating vasomotor action similar to that of the RVLM. Furthermore, the DM, although not as powerful as the RVLM in pressor action, contributes also to the maintenance of the resting systemic arterial pressure (SAP). Information, however, is lacking whether the CVLM can effect sympathetic inhibition through the DM. An attempt to answer this inquiry is the first objective of the present study.

The paramedian reticular nucleus (PRN) in the medulla is also an area effecting inhibition over sympathetic-pressor actions. Simultaneous activation of PRN subsequently eliminates the pressor action induced by DM or RVLM activation (Yang et al., 1992). It also stops and decreases the SAP rise during the course of asphyxia (Yang et al., 1992). Since both the PRN and CVLM effect sympathoinhibition, the second objective of the present paper is to study the interrelationship between the PRN and CVLM.

\section{Materials and methods}

Experiments were carried out in 56 adult cats of either sex weighing 2.3-3.6 kg, anesthetized intraperitoneally with a mixture of $\alpha$-chloralose $(40 \mathrm{mg} / \mathrm{kg}$ ) and urethane $(400 \mathrm{mg} / \mathrm{kg})$, and paralyzed with gallamine triethiodide (2 $\mathrm{mg} / \mathrm{kg} / 30 \mathrm{~min})$. This regimen produced a stable anesthesia for at least $8 \mathrm{~h}$, as indicated by a steady level of blood pressure and heart rate (HR). The femoral vein was cannulated for infusion. After the trachea was intubated, the animal was artificially ventilated with the end tidal $\mathrm{CO}_{2}$ maintained at approximately $4 \%$. The femoral artery was cannulated for measurement of the SAP through which the mean MSAP, indirect cardiac contractile force index $(\mathrm{d} p / \mathrm{d} t)$ and HR were integrated, as have been described previously (Chai et al., 1988; Lin et al., 1989). All recordings were made on a Gould $1000 \mathrm{~S}$ polygraph. The rectal temperature was kept at $37 \pm 1^{\circ} \mathrm{C}$ by a homeostatic blanket (Harvard).

Brain stimulations were carried out by a Kopf stereotaxic instrument (David-Kopt Instruments, Tujunga, CA, USA) while the animals were held in a prone position as described previously (Chai et al., 1988; Lin et al., 1989). The brain regions included the sympathoexcitatory DM and RVLM and the sympathoinhibitory CVLM and PRN. Stimulations were accomplished through a three-barrel micropipette (World Precision Instruments) prepared through a modified Naishige puller (Wu et al., 1995). The micropipettes were inserted into the brain at an angle of $34^{\circ}$. The tip of each capillary was $20-40 \mu \mathrm{m}$. One pipette containing physiological saline inserted with a piece of silver wire was used for electrical stimulation. A constant current of $50-100 \mu \mathrm{A}$ of rectangular pulses (in $15 \mathrm{~s}$ train, $0.5 \mathrm{~ms}$ duration and $80 \mathrm{~Hz}$ ) was generated from a Grass S-88 stimulator coupled with a constant current unit. This same pipette was also used for electrolytic lesion by means of direct current (DC, $1 \mathrm{~mA}$ for $50 \mathrm{~s}$ ) from the same stimulator. Another pipette was filled with Glu (0.25 M in artificial CSF with $1 \%$ pontamine sky blue, $\mathrm{pH}$ 7.4) for stimulation $(70 \mathrm{nl})$, while the remaining pipette was filled with kainic acid (KA, $24 \mathrm{mM}$ in artificial CSF with $1 \%$ pontamine sky blue, $\mathrm{pH}$ 7.4) for lesioning (200 nl). The latter two pipettes were connected through two separate PE-50 tubings, each to a pneumatic pump (Medical System, BH-2) for injection. Based on postmortem histological examination as shown by the stain of pontamine blue, the size of area affected by microinjection of $70 \mathrm{nl}$ was $0.15 \mathrm{~mm}$ in diameter and of $200 \mathrm{nl}$ was $0.22 \mathrm{~mm}$ in diameter. 


\subsection{Recording of nerve activities}

Preparation of the left vertebral sympathetic nerves and recording of its activities through the upper thorax has been previously described (Chai et al., 1993a,b). In brief, under an operation microscope, the nerve was dissected free from the surrounding connective tissues, desheathed, and cut at the distal end. A conventional electrophysiological setup and a bipolar platinum electrode were used to record and amplify the efferent whole nerve activities (bandpass: 10-3 kHz). The signals were monitored with an oscilloscope (Tektronix 5113) and stored on a tape recorder (Neuro Data DR-886) for later analysis. Whole nerve activities were rectified, and integrated by an integrator (Gould 13-4615-70) with a reset time of 1 or $5 \mathrm{~s}$. To acquire the actual neural activity, the noise level of the records obtained $10 \mathrm{~min}$ after the animal's death was subtracted from the whole nerve activity (Yen et al., 1991).

\subsection{Histology}

At the end of each experiment, the animal was sacrificed by an i.v. bolus of saturated $\mathrm{KCl}$. Frozen sections of the brain, $50 \mu \mathrm{m}$ in thickness, were cut by a cryostat microtome (Reichert-Jung, 2800 Frigocut) and stained with Cresyl violet. Sites of chemical stimulation and lesion were reconstructed from sections containing the electrode tracks and marks of the pontamine sky blue.

\subsection{Data analysis}

Percent changes of SAP and perturbation of nerve activity in response to microinjection of $\mathrm{Glu}$ into the sympathoexcitatory or sympathoinhibitory sites were calculated by dividing the value of maximum change with the control value, (Response value - Control value)/(Control value) $\times 100 \%$. The control value of nerve activity was acquired by averaging the integrated nerve activities from six consecutive recordings for $5 \mathrm{~s}$ before stimulation. The largest value deviated from the control level at the period $30 \mathrm{~s}$ after the application of stimulation was considered as the value of maximum change, which was positive during excitation and negative during inhibition of the nerve activity. Percent changes of responses were grouped and classified according to the preparation of animals and injection sites. Data were presented as mean \pm S.E.M. $P$ value less than 0.05 calculated from Student's $t$-test was considered to be statistically significant.

Activation of baroreceptor reflex. Baroreceptor reflex was activated by phenylephrine $24 \mu \mathrm{g} / \mathrm{kg}$, i.v. Effects of baroreceptor reflex were expressed by the barosensitive value (Cerutti et al., 1994).

$$
\begin{aligned}
\text { Barosensitive value }= & \frac{\Delta \text { Heart rate }}{\Delta \text { Mean systemic arterial pressure }} \\
& \times(\text { beats } / \mathrm{mmHg} \cdot \min )
\end{aligned}
$$

\section{Results}

\subsection{Mediation of the CVLM-depressor effect through the $D M$}

Effects of lesioning the DM with KA on the depressor responses evoked by Glu injection into the CVLM were studied in a total of 17 animals. In 11 of these animals, bilateral lesions were made, first in the DM ipsilateral to the CVLM then followed by the remaining (contralateral) DM. In the other 6 animals the procedure of DM lesioning were reversed, i.e. first contralateral then ipsilateral. The effects of lesioning are illustrated in Table 1 and Fig. 1. Lesioning the DM on one side, regardless whether ipsilateral or contralateral, substantially decreased the resting MSAP; from 113 to $94 \mathrm{mmHg}(-19 \mathrm{mmHg}, n=11)$, or from 120 to $86 \mathrm{mmHg}(-34 \mathrm{mmHg}, n=6)$. The resting MSAP, however, did not further decrease following bilateral DM lesions (Table 1).

Comparing the depressor responses of the CVLM induced by Glu, it was found that regardless of which side (ipsilateral or contralateral) of the DM was lesioned first, the CVLM induced-decreases in both SAP and VNA were significantly attenuated. Attenuation of these two components was similar. After both DM were lesioned, the Glu-induced depressor responses of the CVLM were almost eliminated. The MSAP fell only 3\% compared to a fall of $39 \%$ before lesioning, while VNA did not change at all as compared to a decrease of $35 \%$ (Table 1).

The barosensitivity to phenylephrine $24 \mu \mathrm{g} / \mathrm{kg}$, i.v. in 15 animals before and after unilateral DM lesion was compared. Before lesioning it was 2.21 beats $/ \mathrm{mmHg} \cdot \mathrm{min}$; after lesioning, it was reduced to 0.96 beats $/ \mathrm{mmHg} \cdot \mathrm{min}$.

\subsection{Mediation of the CVLM-depressor effect through the RVLM}

In 24 cats, effects of RVLM lesioning on the Glu-induced depressor responses of the CVLM were studied. The effects are summarized in Table 1 and illustrated in Fig. 2. Among these 24 cats, 17 were subjected to a RVLM lesion first on the same side of the CVLM stimulation, then followed by a lesion on the contralateral side. Lesioning the RVLM also reduced the resting MSAP.

Lesioning the RVLM, regardless of which side, produced a significant decrease of the Glu-induced depressor responses by the CVLM, including decreases of MSAP and VNA. The extent of the decrease was large even with unilateral lesioning. A MSAP decrease caused by the CVLM activation $(-40 \%)$ was reduced to $-4 \%$ after the RVLM lesioning. This is $90 \%$ attenuation of the response. Similarly, a decrease in VNA $(-33 \%)$ was reduced to $-3.9 \%$, a $88 \%$ attenuation by the RVLM lesion. Compared to this, unilateral lesioning of the DM caused MSAP attenuation of $67 \%(-40 \%$ to $-10 \%)$, and VNA attenua- 
Table 1

Effects of DM or RVLM lesion by KA on the depressor responses of CVLM induced by Glu

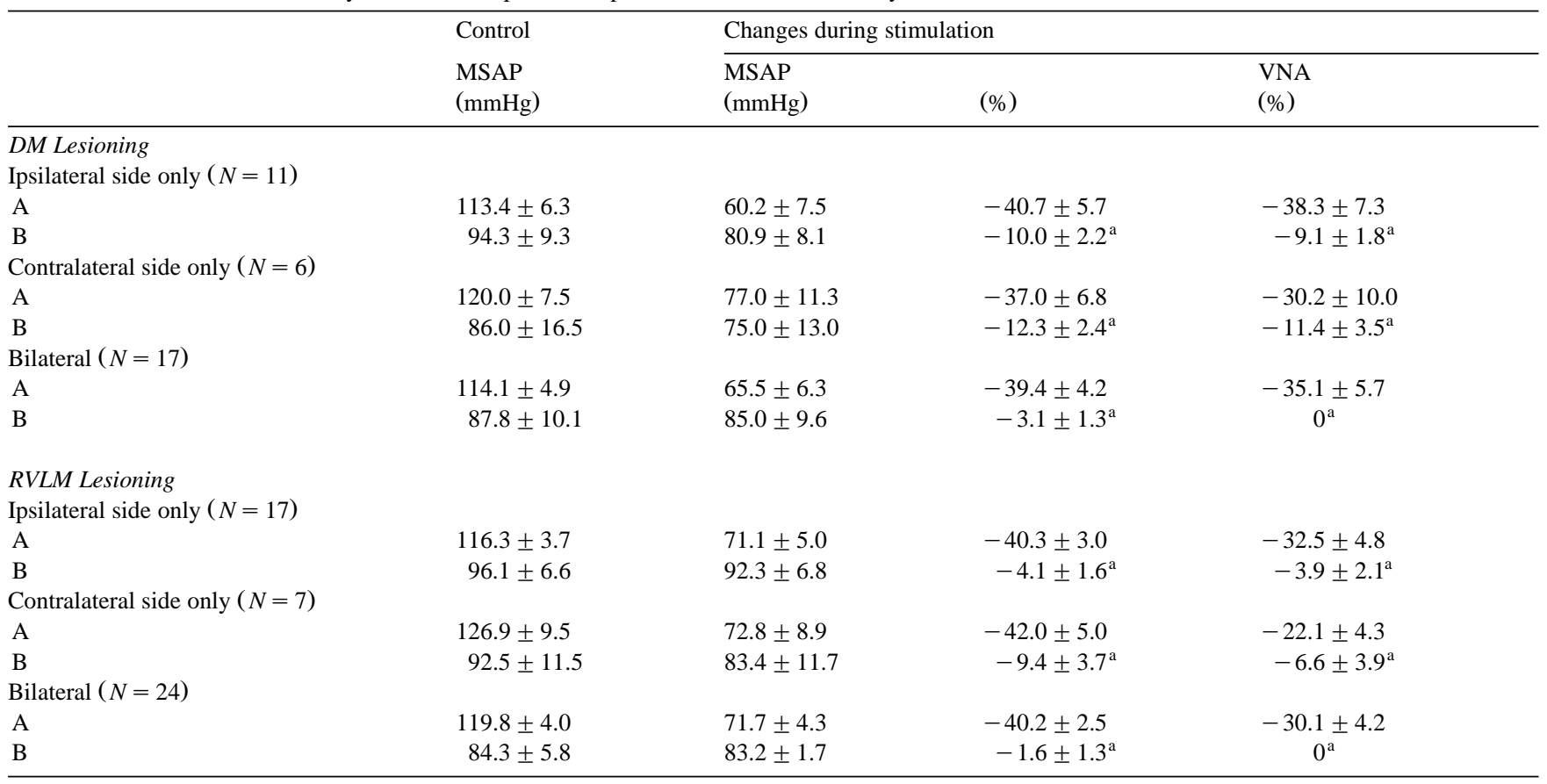

$N$, number of cats; values, mean $\pm \mathrm{SE}$; ${ }^{a}$ Values are statistically significant by Student's $t$-test with $P<0.01$.

A, before lesioning; $\mathrm{B}$, after lesioning.

\section{Cat 030796}

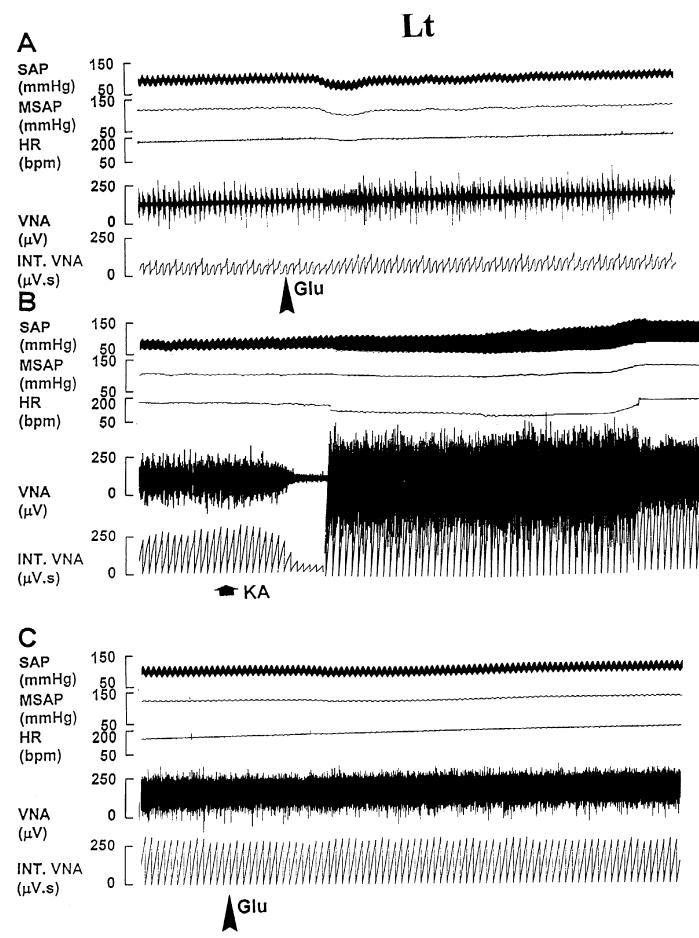

Rt

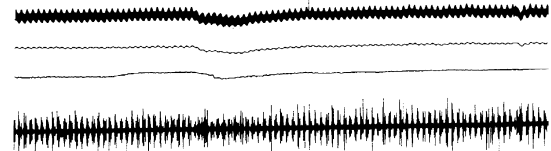

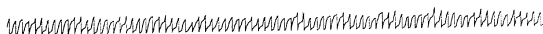
$\Lambda^{\text {Glu }}$

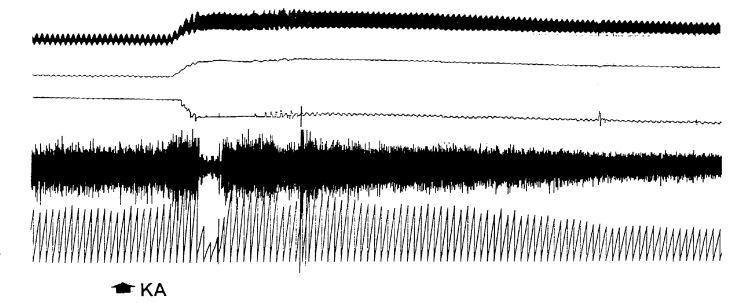

- KA
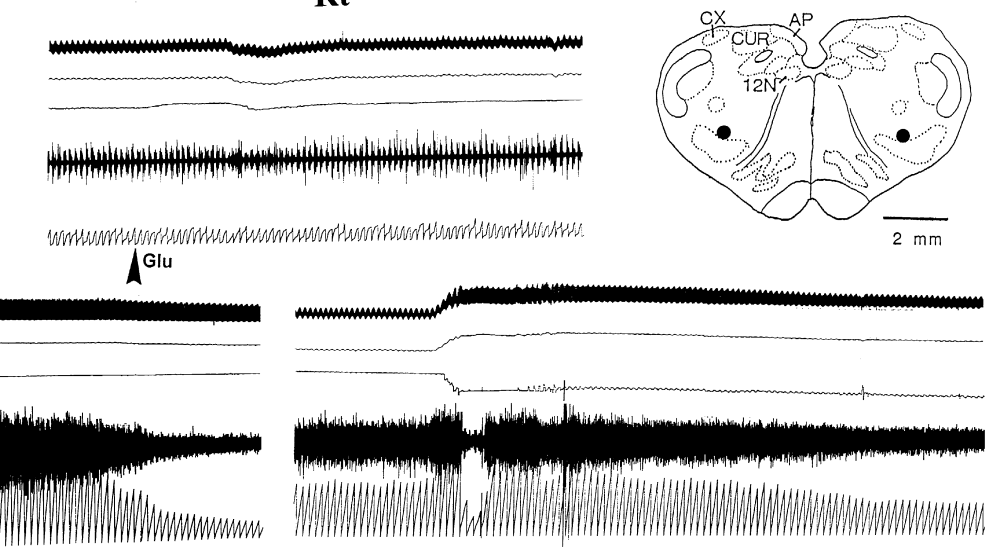
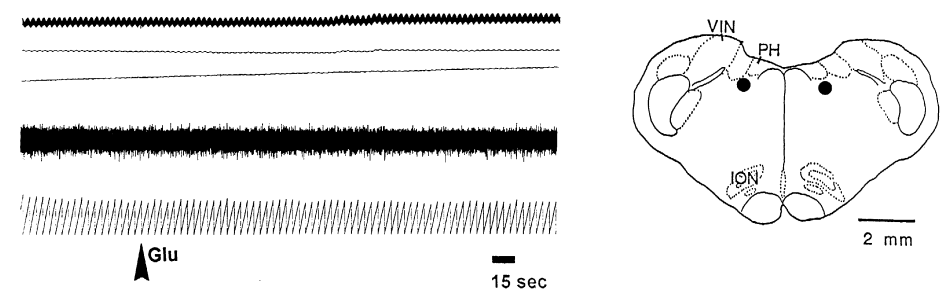

Fig. 1. (Cat 030796). The depressor responses of the CVLM induced by Glu were markedly reduced after lesioning the DM. Upper panels show that microinjection of Glu $(0.25 \mathrm{M}, 70 \mathrm{nl})$ into either the left (Lt) or right (Rt) CVLM produced decreases in SAP, VNA and HR. KA (24 mM, $200 \mathrm{nl})$ was microinjected into the same point of DM on both sides, first on the Lt then on the Rt, at an interval of $2 \mathrm{~h}$. Note the marked pressor responses after KA in middle panels. Lower panels show that repeated microinjection of a same dose of Glu into the same points in CVLM ipsilateral to the lesioned site, each at $2 \mathrm{~h}$ after KA lesioning, produced a much reduced depressor response. In this and the following figures, all kinds of arrow indicate the time of giving microinjection. The coronal brain sertions of the right show sites (indicated by black dots) for Glu and KA injections in the CVLM (upper) and the DM (lower). CVLM, caudal ventrolateral medulla; KA, kainic acid; SAP, systemic arterial pressure; VNA, vertebral nerve activities. 
Cat 031395

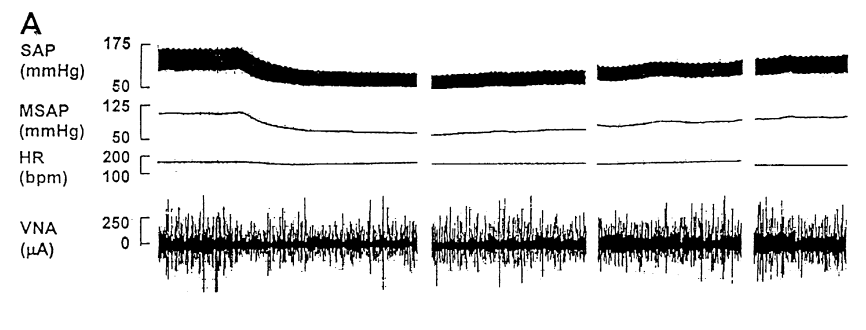

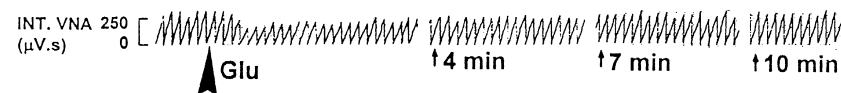
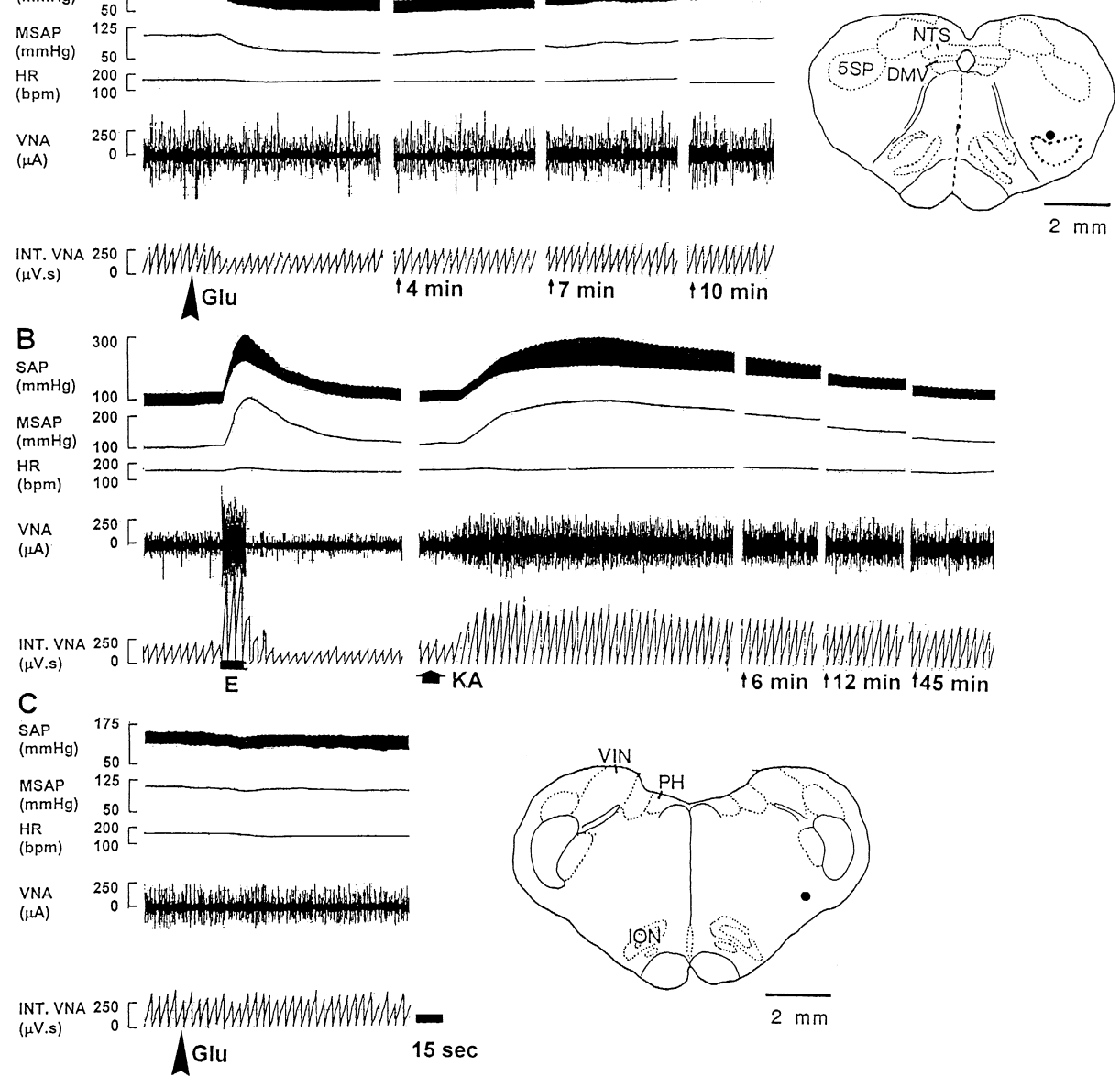

Fig. 2. (Cat 031395). The depressor responses of the CVLM induced by Glu were markedly reduced after lesioning the ipsilateral RVLM. (A) Microinjection of Glu $(0.25 \mathrm{M}, 70 \mathrm{nl})$ into a point in the CVLM (shown in the upper histological section) produced decreases of SAP and VNA with little change in the HR. (B) A point in the RVLM (shown in the bottom histological section) was localized first by electrical stimulation (100 $\mu$ A, $80 \mathrm{~Hz})$ then by microinjection of KA ( $24 \mathrm{mM}, 200 \mathrm{nl}$ ) into the same point. Note the marked (SAP increase to $300 \mathrm{mmHg}$ ) and prolonged (45 min) pressor responses in both SAP and VNA. (C) Two hours after RVLM lesioning, the same dose of Glu into the same point in the CVLM produced a much reduced depressor response.

tion of $75 \%(-38 \%$ to $-9 \%)$ (Table 1). Thus, a reduction of CVLM-induced responses after DM lesion was less than that after RVLM lesion. After bilateral RVLM lesioning, the Glu-induced depressor responses from the CVLM were almost eliminated (Table 1).

In 23 animals the barosensitivity tested by intravenous phenylephrine ( $24 \mu \mathrm{g} / \mathrm{kg}$ ) was compared before and after unilateral RVLM lesioning by KA. The control barosensitivity was 2.03 beats $/ \mathrm{mmHg} \cdot \mathrm{ms}$. After lesioning, it was reduced to 0.43 beats $/ \mathrm{mmHg} \cdot \mathrm{min}$, significantly more pronounced than that after DM lesioning.

\subsection{PRN and CVLM depressor effects}

\subsubsection{PRN lesioning}

The depressor PRN regions is known to be less sensitive to excitatory amino acid (Chai et al., 1990). Therefore, besides KA, the PRN was also lesioned by DC current. Table 2 and Fig. 3 summarize the results.

3.3.1.1. KA lesioning. Lesioning PRN with KA ipsilateral $(N=4)$ to the point of the CVLM receiving Glu activation produced essentially no change in the resting MSAP. However, the CVLM depressor effects were significantly attenuated from $-46 \%$ to $-35 \%$ and VNA from a control $-29 \%$ to $-21 \%$.

Similarly, lesioning the contralateral PRN by KA produced a significant attenuation of the CVLM-induced decrease in MSAP, but the induced decrease in VNA was only slightly, but not significantly attenuated.

3.3.1.2. DC lesioning. DC lesion of the PRN produced a more consistent attenuation of the Glu-induced depressor responses of the CVLM. In the experiments of electrolytic 
Table 2

Effects of PRN lesion by KA or DC electrolysis on the depressor responses of CVLM induced by Glu

\begin{tabular}{|c|c|c|c|c|}
\hline & \multirow{2}{*}{$\begin{array}{l}\text { Control } \\
\text { MSAP } \\
(\mathrm{mmHg})\end{array}$} & \multicolumn{3}{|c|}{ Changes during stimulation } \\
\hline & & $\begin{array}{l}\text { MSAP } \\
(\mathrm{mmHg})\end{array}$ & $(\%)$ & $\begin{array}{l}\text { VNA } \\
(\%)\end{array}$ \\
\hline $\mathrm{A}$ & $111.5 \pm 6.4$ & $60.5 \pm 8.2$ & $-46.4 \pm 9.6$ & $-28.9 \pm 6.1$ \\
\hline $\mathrm{B}$ & $113.0 \pm 4.6$ & $74.5 \pm 8.1$ & $-34.6 \pm 5.0^{\mathrm{a}}$ & $-20.8 \pm 5.7^{\mathrm{a}}$ \\
\hline B & $114.4 \pm 9.9$ & $86.9 \pm 8.7$ & $-23.5 \pm 6.9^{\mathrm{a}}$ & $-20.1 \pm 10.5$ \\
\hline \multicolumn{5}{|c|}{ PRN DC Lesioning } \\
\hline \multicolumn{5}{|c|}{ Ipsilateral side only $(N=8)$} \\
\hline A & $112.2 \pm 4.3$ & $66.6 \pm 6.9$ & $-41.4 \pm 4.6$ & $-26.9 \pm 5.2$ \\
\hline $\mathrm{B}$ & $108.8 \pm 6.8$ & $80.6 \pm 9.8$ & $-27.2 \pm 5.7^{\mathrm{a}}$ & $-12.3 \pm 5.3^{\mathrm{a}}$ \\
\hline A & $112.5 \pm 4.4$ & $67.5 \pm 5.4$ & $-40.3 \pm 3.7$ & $-26.9 \pm 3.3$ \\
\hline $\mathrm{B}$ & $109.8 \pm 5.3$ & $86.3 \pm 9.3$ & $-22.1 \pm 6.4^{\mathrm{a}}$ & $-4.5 \pm 1.7^{\mathrm{a}}$ \\
\hline
\end{tabular}

$N$, number of cats; value, mean $\pm \mathrm{SE} ;{ }^{a}$ Values are statistically significant by Student's $t$-test with $P<0.05$.

$\mathrm{A}$, before lesioning; B, after lesioning.

lesion made to PRN either first in the ipsilateral $(N=8)$ or contralateral $(N=8)$ side, the CVLM-elicited decreases of MSAP and VNA were significantly attenuated. The reduction of VNA was more apparent after DC lesioning $(-22.4 \%)$ than after KA lesioning. After bilateral electrolytic lesions in the PRN, the reduction of depressor responses induced by Glu in the CVLM were greater for VNA, but not for MSAP.

\subsubsection{CVLM lesioning}

In 16 cats, the depressor responses caused by electrical stimulation of the PRN were compared before and after CVLM lesioning by KA. In eight of these animals, lesions were made first on the ipsilateral, then followed by the remaining CVLM. In the remaining eight animals, the initial side of lesioning was reversed. Table 3 and Fig. 4 illustrate the results. The resting MSAP was essentially not altered following KA lesions on the CVLM regardless of ipsilateral or contralateral to the side of PRN stimulation or after bilateral KA lesions. After lesioning one side of the CVLM, the PRN-responses, both decreases in MSAP ( -21 to $-22 \%$ against the control -37 to $-34 \%$, a reduction of $1-3 \%)$ and VNA ( -39 to $-60 \%$ against the control -56 to $-74 \%$, a reduction of $21-18 \%$ ) were slightly but significantly reduced. Marked attenuation of the PRN-responses were observed only after bilateral CVLM lesions. The CVLM-induced MSAP decrease was attenuated by $43 \%$ (from the control $-35 \%$ to $-20 \%$ ), while that of VNA decrease was 56\% (from the control $-62 \%$ to $-27 \%$ ) (Table 3).

\section{Discussion}

Major findings of the present experiment are as follows: I). Decreases of SAP and sympathetic nerve activity consequent to Glu activation of the CVLM and the barosensitivity to intravenous phenylephrine were attenuated following KA lesion on either DM or RVLM. The attenuation was slightly greater after RVLM than DM lesion. II). The depressor CVLM and PRN regions are mutually associated. Lesioning either one decreased the depressor responses caused by activation of the other. CVLM lesioning produced slightly greater effect than that of PRN lesioning.

\subsection{Methodology}

In the present study, KA was used as a tool for lesioning, taking advantage that KA affects neuronal cell bodies and dendrites but not axons, thus sparing the passing fibers around the injection site (Goodchild and Dampney, 1985; Olney, 1978). By applying $24 \mathrm{mM} \mathrm{KA}$ the damage to the tissue was rather fast. Thirty min after KA application to the DM, RVLM or CVLM, a 80-90\% loss of the Glu-induced responses from the same region was observed. In the present study we compared the CVLM-induced depressor responses at $1 \mathrm{~h}$ and $2 \mathrm{~h}$ after KA application and came to a conclusion that the damage to the tissue was complete by $2 \mathrm{~h}$ after lesioning. 


\section{Cat 092095}

A 200 [ 20

$\underset{\text { (mmHg) }}{{ }_{2}}$

$\underset{(\mathrm{mmHg})}{\operatorname{MSAP}}{ }_{50}^{150}[$

$\underset{\text { (bpm) }}{\mathrm{HR}} \quad \stackrel{200}{-50}[$
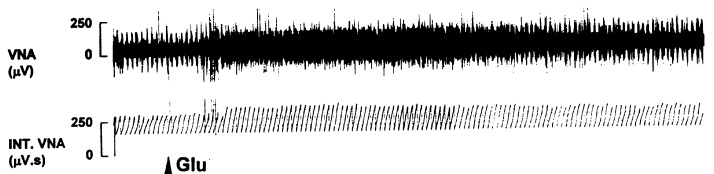

$\Lambda^{\text {Glu }}$
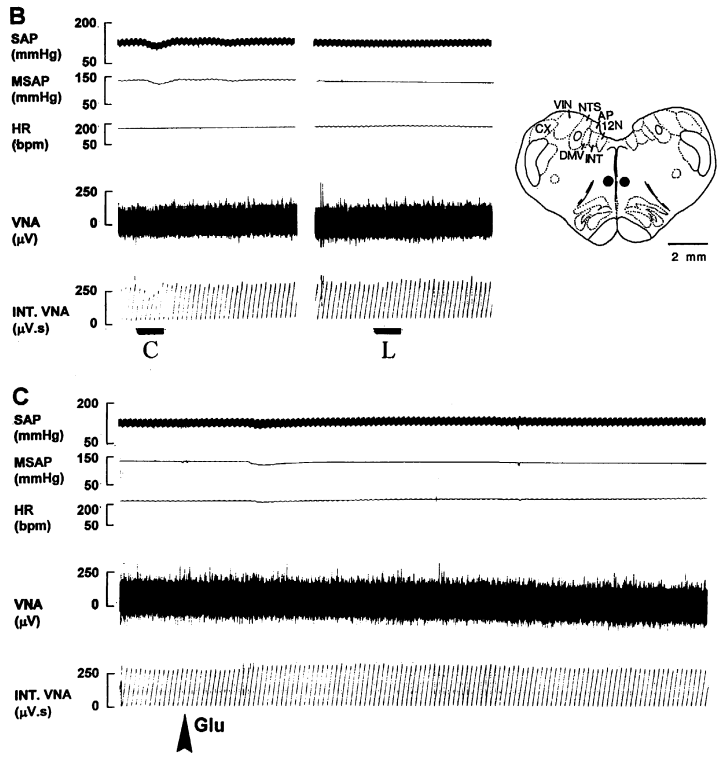

Rt

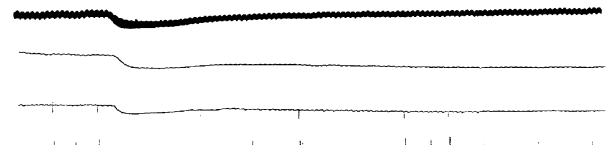

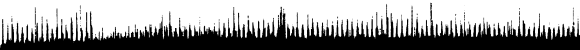

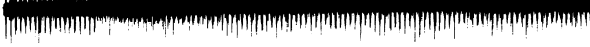

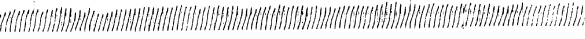

$\Lambda^{\text {Glu }}$
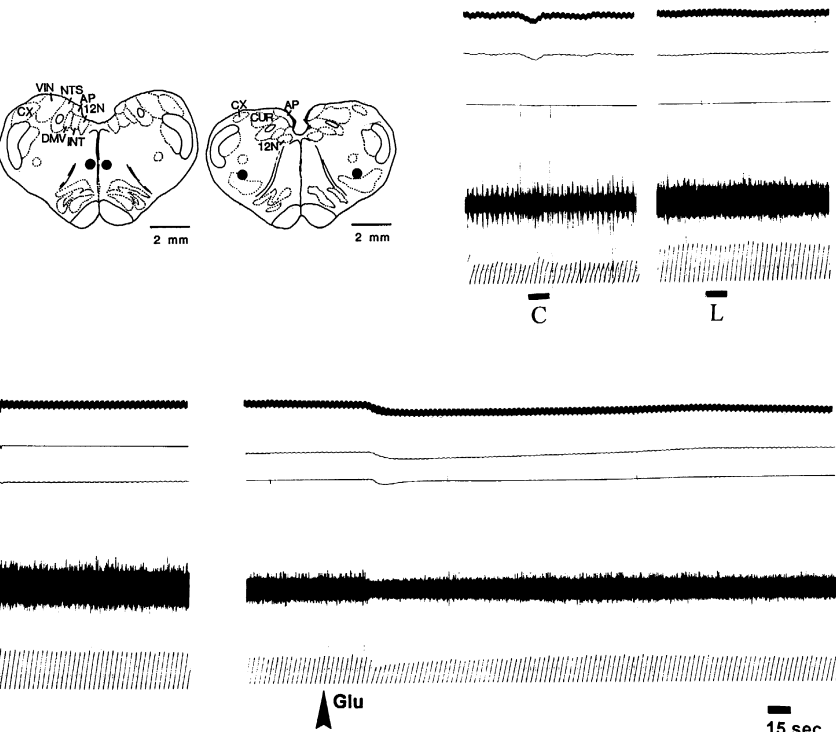

Fig. 3. (Cat 092095). The Glu-induced depressor responses of the CVLM were slightly reduced after DC electrolytic lesion of the PRN. (A) Microinjection of Glu $(0.25 \mathrm{M}, 70 \mathrm{nl})$ into a point either in the left (Lt) or right (Rt) CVLM (sites of injection and shown in the histological section on the right) produced depressor responses in decreases of SAP, VNA and HR. (B) Two points in the Lt and Rt PRN (indicated in the left histological section) were localized by electrical stimulation $(100 \mu \mathrm{A})$ followed by DC electrolytic lesion $(1 \mathrm{~mA}, 50 \mathrm{~s})$ in the same point. Note the electrical induced-responses in both PRNs after lesioning (L) were much reduced in comparison with those of the control (c). (C) After PRN lesioning, depressor responses induced by a same dose of Glu in either CVLM ipsilateral to the PRN lesioned site, particularly that of the Lt, were reduced. PRN, paramedian reticular nucleus.

Table 3

Effects of CVLM lesion by KA on the electrically induced depressor responses of PRN

\begin{tabular}{|c|c|c|c|c|}
\hline & \multirow{2}{*}{$\begin{array}{l}\text { Control } \\
\text { MSAP } \\
(\mathrm{mmHg})\end{array}$} & \multicolumn{3}{|c|}{ Changes during stimulation } \\
\hline & & $\begin{array}{l}\text { MSAP } \\
(\mathrm{mmHg})\end{array}$ & $(\%)$ & $\begin{array}{l}\text { VNA } \\
(\%)\end{array}$ \\
\hline A & $96.3 \pm 4.5$ & $61.3 \pm 3.9$ & $-36.6 \pm 2.0$ & $-56.1 \pm 6.6$ \\
\hline B & $101.0 \pm 3.6$ & $79.2 \pm 4.2$ & $-21.2 \pm 3.8^{\mathrm{a}}$ & $-39.3 \pm 9.8^{a}$ \\
\hline \multicolumn{5}{|c|}{ Contralateral side only $(N=8)$} \\
\hline B & $99.1 \pm 8.4$ & $78.8 \pm 9.3$ & $-21.5 \pm 3.7^{\mathrm{a}}$ & $-60.3 \pm 8.7^{\mathrm{a}}$ \\
\hline \multicolumn{5}{|c|}{ Bilateral $(N=16)$} \\
\hline A & $98.1 \pm 3.4$ & $63.6 \pm 3.2$ & $-35.4 \pm 1.9$ & $-61.51 \pm 5.6$ \\
\hline B & $77.8 \pm 3.9$ & $63.1 \pm 5.0$ & $-19.6 \pm 2.3^{\mathrm{a}}$ & $-26.6 \pm 5.5^{\mathrm{a}}$ \\
\hline
\end{tabular}

$N$, number of cats; values, mean $\pm \mathrm{SE} ;{ }^{a}$ Values are statistically significant by Student's $t$-test with $P<0.01$.

A, before lesioning; $\mathrm{B}$, after lesioning. 


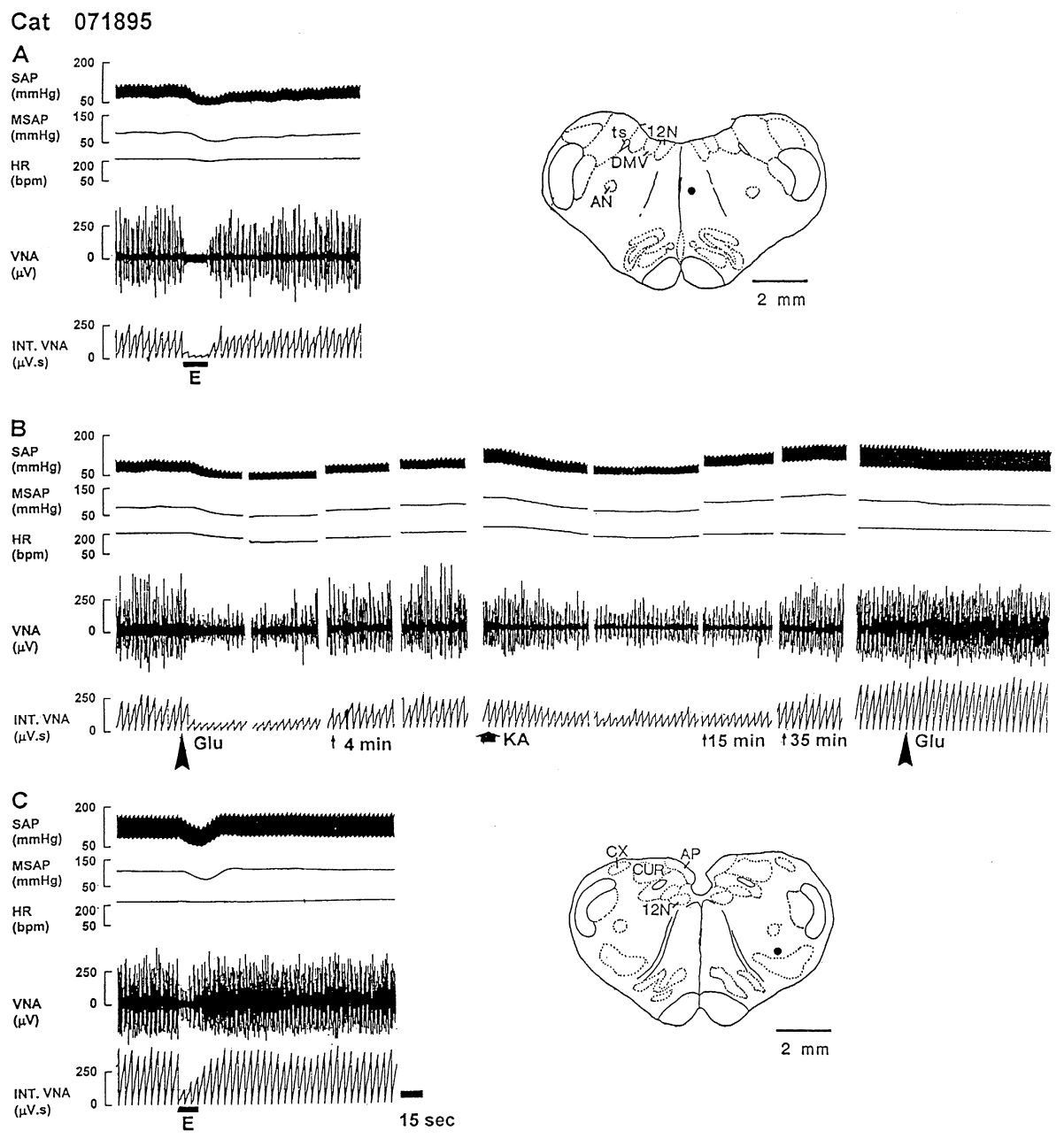

Fig. 4. (Cat 071895). The PRN depressor responses were slightly attenuated by KA lesioning of the ipsilateral CVLM. (A) Control responses of the PRN induced by electrical stimulation of the PRN. (B) A point in the CVLM was localized first by electrical current (not shown) and then Glu (0.25 M, $70 \mathrm{nl}$ ). The same point in the CVLM (indicated in the bottom right histological section) and the nearby region were lesioned by microinjection of KA (24 mM, $200 \mathrm{nl}$ ). Note that KA produced similar decreases in SAP and VNA as Glu but with much longer duration. The last segment show that $2 \mathrm{~h}$ after KA lesioning, the same dose of Glu in the same point of the CVLM produced very little responses. After CVLM lesioning the depressor responses of the PRN, the duration of decreases in both SAP and VNA, were much shortened, but the magnitude of decrease reduced only slightly.

It is necessary to emphasize that in our work the effects of microinjections of Glu $(0.25 \mathrm{M}, 70 \mathrm{nl})$ to the DM, RVLM and CVLM were always reproducible when they were repeated at an interval of 30-40 min over a period of $2 \mathrm{~h}$ or longer.

\subsection{The CVLM produces decreases of systemic arterial} pressure and sympathetic nerve activity via inhibition of the sympathetic-pressor mechanism of the RVLM

A considerable number of studies have shown that decreases of SAP and sympathetic activity elicited by activation of the CVLM are mediated through inhibition of the pressor neurons in the RVLM. For instance, inactivation of RVLM neurons with muscimol, a GABA agonist, reduced the decreases of SAP and the sympathetic splanchnic nerve activity induced by microinjection of Glu into the CVLM (Willette et al., 1984a,b). One of the drawbacks of using a single agonist or antagonist to inactivate the RVLM is a reduction of the baseline resting SAP. When a stable resting SAP was maintained despite inactivation of the RVLM vasomotor response by simultaneous microinjections of GABA agonist and antagonist into the RVLM, virtually all vasomotor effects normally elicited from the CVLM were abolished (Blessing, 1988). In addition, in baroreceptor denervated rats, chemical lesioning of the CVLM with KA produced a sustained rise of SAP and particularly a greater rise (200\%) of sympathetic splanchnic nerve activity, suggesting tonic influence from the CVLM to effect sympathoinhibition (Cravo and Morrison, 1993). These studies echo other experiments (Blessing and Reis, 1982; Li et al., 1991), and suggest the existence of the tonically active CVLM that effects sympathetic inhibition. Inhibition is mediated indirectly through the RVLM, inasmuch as there is no concrete anatomical finding showing a direct projection pathway from the CVLM to the 
preganglionic sympathetic neurons of the spinal cord (Amendt et al., 1979).

\subsection{The CVLM serves as an relay station to mediate the baroreceptor reflex}

A large number of experiments also have demonstrated that decreases of SAP and sympathetic nerve activity consequent to baroreceptor activation is mediated through an intermediate pathway in the CVLM before reaching the final neuron pools of the RVLM. For instance, Agarwal et al. (1990) have shown that microinjection of kynurenic acid, a broad spectrum Glu antagonist, into the ipsilateral CVLM blocked the inhibitory response of the sympathetic activity due to a rise of SAP by intravenous phenylephrine, and attenuated the inhibitory response of the RVLM neurons by $43 \%$. Activation of the CVLM by muscimol, a GABA agonist, eliminated the SAP decrease induced by electrical stimulation of the aortic nerve (Gordon et al., 1987). Separate measurements of latencies of the CVLM unit firing showed, that the sum of the latency following an orthodromic activation of the aortic nerve, and that following an antidromic stimulation of the RVLM, was about equal to the latency of the RVLM unit firing activated by aortic nerve stimulation (Terui et al., 1990). Thus, a functional baroreceptor loop passing through the CVLM is suggested.

\subsection{The CVLM mediates its systemic depressor and baroreflex actions not only through the RVLM but also the $D M$}

The present study observed that not only the RVLM, but also the DM is involved in the CVLM depressor responses induced by Glu. We found that following acute KA lesioning of the pressor DM ipsilateral to CVLM activation, the Glu induced-decreases of SAP and VNA were significantly reduced. The reduction of the SAP decrease was more apparent after the remaining DM was also chemically lesioned. It is noteworthy that the damage resulting from RVLM lesioning is more severe than that of $\mathrm{DM}$ lesioning in terms of eliciting depressor responses from the CVLM. This is also true for baroreceptor-responses induced by intravenous phenylephrine, as shown in the present experiment. After bilateral RVLM lesioning, the reduction of barosensitivity $(0.43$ beats $/ \mathrm{mmHg} \cdot \mathrm{min})$ was greater than that after bilateral DM lesioning (0.96 beats $/ \mathrm{mmHg} \cdot \mathrm{min}$ ). This is consistent with our previous findings that pressor points in the medulla eliciting elevation of MSAP higher than $120 \mathrm{mmHg}$ following microinjection of homocysteic acid was observed only in the RVLM. Again, points eliciting MSAP increases of 80-120 $\mathrm{mmHg}$ was observed more frequently in the RVLM (16\%) than in the DM (3\%) (Lin et al., 1989), while unilateral KA-lesioning of the RVLM was sufficient to produce a pronounced decrease of the resting SAP, while bilateral lesioning of the DM was required to produce an equivalent effect (Su et al., 1989).

\subsection{Contribution of the DM and the RVLM in the mainte- nance of constancy in vasomotor tonus}

The maintenance of constancy in vasomotor tonus is effected mainly through the sympathetic system. Supraspinal neurons of this system involve principally those located in the medulla. These pressor neurons in the medulla are constantly modulated by baroreceptors and to some extent by chemoreceptors. As has been mentioned previously, a population of neurons in the CVLM synapses with the pressor neurons in the medulla to effect inhibition of their functions. The RVLM has been recognized as the most important mechanism effecting vasomotor action (Calaresu, 1988; Ciriello et al., 1986; Dampney et al., 1994). Recently, the existence of neurons in the DM integrating vasomotor function has also been repeatedly demonstrated in cats (Chai et al., 1988, 1990, 1991, 1993a,b, 1997; Lin et al., 1989; Su et al., 1989), rabbits (Goodchild and Dampney, 1985) and rats (Yardley et al., 1989). Functionally, the DM generates part of the $2-6 \mathrm{~Hz}$ rhythm of the sympathetic nerve discharge (Barman and Gebber, 1983). For sometime the pressor action of DM has been considered to be a result secondary to activation of a fiber passage originating from the RVLM (Farlow et al., 1984). Recently, to consolidate this concept, Hirooka et al. (1994) have shown that in rabbits, after inactivation of the RVLM with kynurenic acid, a broad spectrum amino acid antagonist, the Glu-induced vasomotor action in DM was abolished. On the other hand, it has been shown that in rats anesthetized with pentobarbital and chloralose, but not urethane, the reduction of MSAP consequent to DC lesioning of the RVLM could return to $76 \%$ of the pre-lesioned level 30 min after lesioning (Cochrane et al., 1988). We also showed that following application of KA to the DM, the Glu-induced vasopressor action from the RVLM was decreased (Su et al., 1989). This suggests that other vasomotor mechanisms, in which the DM may be a good candidate, probably share with the RVLM for vasomotor integration. In this regard, we have demonstrated that degenerating fibers from the DM to the intermediolateral cell column of the thoracic cord was observed after chronic KA lesioning of the DM (Wang et al., 1996). Besides, axon terminals synapsed with the thoracic preganglionicsympathetic neuron were observed after application of dextran, an anterograde tracer, in the DM (Lan et al., 1997). Yardley et al. (1989) comments that the DM may not be essential for the genesis of vasomotor tone but may be important for supporting the defence responses. Indeed, we have observed that the onset latency of evoked potential recorded in the periaqueductal grey of the midbrain was shorter after DM (3.5 ms) than RVLM (5.6 ms) activation (Yen et al., 1995). In addition, the content of plasma catecholamine, a good index of defence reaction, is 
higher during Glu activation in the DM than in the RVLM (Chai et al., 1991) and the decrease of plasma catecholamine is more marked following KA lesioning of the DM than the RVLM (Chai et al., 1988, 1993a,b; Su et al., 1989). KA lesioning of the DM or RVLM reduced the vasodilatation of the femoral and carotid arteries and vasoconstriction of the splanchnic arteries produced by stimulation of the vocalized defense area of the periaqueductal grey. The reduction of the above arterial responses were greater after DM than RVLM lesioning (Chai et al., 1997).

\subsection{Pressor and depressor regions other than DM, VLM and CVLM}

Recently, the existence of some pressor neurons in the caudalmost ventrolateral medulla is suggested. In cats, microinjection of $500 \mu \mathrm{M}$ Glu into the region just dorsal to the lateral reticular nucleus at the level caudal to the obex produced a distinct pressor response (Iwamoto et al., 1991). In rats, microinjection of Glu (0.25 M) in the ventral surface of the medulla between the rootlets of the hypoglossal and the first cervical nerve produced hypotension. The latter effect was found to be dependent on the integrity of the pressor RVLM (Possas et al., 1994). It is not known whether the CVLM also exerts inhibition on the caudal-most pressor areas.

A depressor mechanism sensitive to excitatory amino acid has been described in the fastigial nucleus of the cerebellum (Chida et al., 1986). The depressors action of the CVLM, however, is independent of this cerebellar mechanism, as the CVLM-depressor responses persist after cerebellectomy (Dembowsky et al., 1989). Activation of neurons in raphe nucleus (pallidus and obscurus) at the level caudal to the obex also produced depressor and sympathoinhibitory responses, similar to that of CVLM activation (Coleman and Dampney, 1995). Different from the CVLM, raphe-inhibition was not affected by neuroinhibitory compounds, GABA, or muscimol. The authors thus suggest that raphe only exerts phasic but not tonic influence on the sympathetic pressor mechanism (Coleman and Dampney, 1995).

\subsection{Correlation between paramedian reticular nucleus (PRN) and CVLM}

The PRN exerts potent inhibition over both the sympathetic pressor and somatic spinal reflex (Chai et al., 1988, 1990; Lin et al., 1988). The inhibition is so strong that during the course of asphyxia when the rise of SAP has reached its maximum, electrical activation of the PRN at this time is still able to reduce the SAP (Yang et al., 1992). One of the drawbacks of PRN activation is its low sensitivity to Glu, though microinjection of homocysteic acid, a more potent excitatory amino acid, can provoke a decrease of the resting SAP and decrease of the spinal reflex; the evoked potential recorded at the ventral root by intermit- tent stimulation of the sciatic afferents (Chai et al., 1990). On the contrary, neurons of the CVLM respond very well to Glu. One may consider that part of the CVLM-inhibitory effect is mediated through the PRN. It is also possible that neurons from the PRN may synapse with neurons in the CVLM to effect sympathetic inhibition. Findings of the present experiment show that both directions are possible, as depressor actions of the CVLM and PRN are mutually affected; acute KA lesioning of the CVLM decreased the depressor responses induced by PRN stimulation, while PRN lesioning decreased the CVLM-induced depressor responses. After lesioning (bilateral) either the PRN or CVLM, the induced VNA reduction following PRN or CVLM activation was abolished. The reduction of SAP decrease induced by PRN activation appears slightly greater after CVLM lesioning than that of the SAP fall induced by CVLM activation after PRN lesioning. This suggests that the CVLM carries more weight in the interrelationship between the CVLM and the PRN. This inhibitory pathway, however, is far less potent than that between the CVLM and RVLM or between the CVLM and DM, as a much greater loss of the CVLM-induced hypotensive action resulted after RVLM or DM lesioning.

In conclusion, findings of our experiments show that decreases of SAP and VNA induced by Glu activation of the CVLM are mediated by both the RVLM and the DM with slight predominance of the former. The inhibitory action by the CVLM and PRN are mutually related. The deficit is more apparent after CVLM than PRN lesioning.

\section{Acknowledgements}

The authors express their gratitude to Drs K.K. Wu and T.C. Lee for their encouragement and support during this study. We thank Mr G.T. Chen for preparation of illustrations and Ms J.J. Pan for preparation of the manuscript. Thanks are due to Dr C.V. Weaver, Professor of Fu Jen Catholic University for comments of this paper. This study was support in part by the Foundation of Biomedical Sciences, Shih-Chun Wang Research Fund and the National Science Council, R.O.C., No. NSC 86-2745-B-001003.

\section{References}

Agarwal, S.K., Gelsema, A.J., Calaresu, F.R., 1990. Inhibition of rostral VLM by baroreceptor activation is relayed through caudal VLM. Am. J. Physiol. 258, R1271-R1278.

Aicher, S.A., Kurucz, O.S., Reis, D.J., Milner, T.A., 1995. Nucleus tractus solitarius efferent terminals synapse on neurons in the caudal ventrolateral medulla that project to the rostral ventrolateral medulla. Brain Res. 693, 51-63.

Amendt, K., Czachurski, J., Dembowsky, K., Seller, H., 1979. Bulbospinal projections to the intermediolateral cell column; a neuroanatomical study. J. Auton. Nerv. Syst. 1, 103-117.

Badoer, E., Chalmers, J., 1992. Interactions of endogenous opioid and 
excitatory amino acid inputs to the caudal ventrolateral medulla of the rat. Neuropharmacology 31, 857-862.

Badoer, E., McKinley, M.J., Oldfield, B.J., McAllen, R.M., 1994. Localization of barosensitive neurons in the caudal ventrolateral medulla which project to the rostral ventrolateral medulla. Brain Res. 657, 258-268.

Barman, S.M., Gebber, G.L., 1983. Sequence of activation of ventrolateral and dorsal medullary sympathetic neurons. Am. J. Physiol. 14, R438-R447.

Blessing, W.W., Reis, D.J., 1982. Inhibitory cardiovascular function of neurons in the caudal ventrolateral medulla of the rabbit: Relationship to the area containing A1 noradrenergic cells. Brain Res. 253, 161171.

Blessing, W.W., Reis, D.J., 1983. Evidence that GABA and glycine-like inputs inhibit vasodepressor neurons in the caudal ventrolateral medulla of the rabbit. Neurosci. Lett. 37, 57-62.

Blessing, W.W., 1988. Depressor neurons in rabbit caudal medulla act via GABA receptors in rostral medulla. Am. J. Physiol. 254, H686-H692.

Bonham, A.C., Jesek, I., 1989. Cardiorespiratory effects of dl-homocysteic acid in caudal ventrolateral medulla. Am. J. Physiol. 256, H688-H696.

Calaresu, F.R., 1988. Medullary basal sympathetic tone. Annu. Rev. Physiol. 50, 511-524.

Cerutti, C., Barres, C., Paultre, C., 1994. Baroreflex modulation of blood pressor and heart rate variabilities in rats: Assessment by spectral analysis. Am. J. Physiol. 266, H1993-H2000.

Chai, C.Y., Lin, R.H., Lin, A.M.Y., Pan, C.M., Lee, E.H.Y., Kuo, J.S., 1988. Pressor responses from electrical or glutamate stimulations of the dorsal or ventrolateral medulla. Am. J. Physiol. 255, R709-R717.

Chai, C.Y., Lin, Y.F., Wang, H.Y., Wu, W.C., Yen, C.T., Kuo, J.S., Wayner, M.J., 1990. Inhibition of spinal reflexes by paramedian reticular nucleus. Brain Res. Bull. 25, 581-588.

Chai, C.Y., Lin, A.M.Y., Su, C.K., Hu, S.R., Yuan, C., Kao, L.S., Kuo, J.S., Goldstein, D.S., 1991. Sympathoadrenal excitation and inhibition by lower brainstem stimulation in cats. J. Auton. Nerv. Syst. 33 35-46.

Chai, C.Y., Chen, S.Y., Wang, S.D., Tseng, C.J., Lin, R.H., Mao, S.P., Horng, H.T., Liu, J.C., Kuo, J.S., 1993a. Precollicular decerebration reduces the pressor responses evoked by stimulation of rostral pons but not medulla in cats. J. Auton. Nerv. Syst. 46, 147-159.

Chai, C.Y., Yuan, C., Horng, H.T., Wang, S.D., Tseng, C.J., Kuo, J.S., 1993b. Neurons in the pressor sites of dorsal and ventrolateral medulla mediate pressor actions of the periaqueductal grey of midbrain. Chin. J. Physiol. 36, 37-45.

Chai, C.Y., Wu, W.C., Hwang, J.J., Wang, M.R., Kuo, J.S., 1997. Attenuation of cardiovascular reactions of vocalized and non-vocalized defence areas of periaqueductal grey following lesions in dorsomedial or ventrolateral medulla of cats. J. Auton. Nerv. Syst. 64, $115-127$

Chida, K., Iadecola, C., Underwood, M.D., Reis, D.J., 1986. A novel vasodepressor response elicited from the rat cerebellar fastigial nucleus: The fastigial depressor responses. Brain Res. 370, 378-383.

Ciriello, J., Caverson, M.M., Polosa, C., 1986. Function of the ventrolateral medulla in the control of the circulation. Brain Res. Rev. 11, 359-391.

Cochrane, K.L., Buchholz, R.A., Hubbard, J.W., Keeton, T.K., Nathan, M.A., 1988. Hypotensive effects of lesions of the rostral ventrolateral medulla in rats are anesthetic-dependent. J. Auton. Nerv. Syst. 22, $181-187$

Coleman, M.J., Dampney, R.A.L., 1995. Powerful depressor and sympathoinhibitory effects evoked from neurons in the caudal raphe pallidus and obscurus. Am. J. Physiol. 268, R1295-R1302.

Cravo, S.L., Morrison, S.F., Reis, D.J., 1991. Differentiation of two cardiovascular regions within caudal ventrolateral medulla. Am. J. Physiol. 261, R985-R994.

Cravo, S.L., Morrison, F., 1993. The caudal ventrolateral medulla is a source of tonic sympathoinhibition. Brain Res. 621, 133-136.
Dampney, R.A.L., 1994. Functional organization of central pathways regulating the cardiovascular system. Physiol. Rev. 74, 323-364.

Day, T.A., Ro, A., Renaud, L.P., 1983. Depressor area within caudal ventrolateral medulla of the rat does not co-respond to the a1 catecholamine cell group. Brain Res. 279, 299-302.

Dembowsky, K., Czachurski, J., Seller, H., 1989. Some properties of the sympathoinhibition from the caudal ventrolateral medulla oblongata in the cat, ciriello. In: Caverson, J., Polosa, M.M.C., (Eds.). Progress in Brain Res. 81, 143-157.

Farlow, D.M., Goodchild, A.K., Dampney, R.A.L., 1984. Evidence that vasomotor neurons in the rostral ventrolateral medulla project to the spinal sympathetic outflow via the dorsomedial pressor area. Brain Res. 298, 313-320.

Feldberg, W., Guertzenstein, P.G., 1976. Vasodepressor effects obtained by drugs acting on the ventral surface of the brain stem. J. Physiol. Lond. 258, 337-355.

Goodchild, A.K., Dampney, R.A.L., 1985. A vasopressor cell group in the rostral dorsomedial medulla of the rabbit. Brain Res. 360, 24-36.

Gordon, F.J., 1987. Aortic baroreceptor reflexes are mediated by NMDA receptors in caudal ventrolateral medulla. Am. J. Physiol. 252, R628R633.

Hirooka, Y., Ploson, J.W., Dampney, R.A.L., 1994. Pressor response from rostral dorsomedial medulla is mediated by excitatory amino acid receptors in rostral VLM. Am. J. Physiol. 267, R309-R315.

Iwamoto, G.A., Brtva, R.D., Waldrop, T.G., 1991. Cardiorespiratory responses to chemical stimulation of the caudalmost ventrolateral medulla in the cat. Neurosci. Lett. 129, 86-90.

Kubo, T., Kihara, M., 1988. Evidence of $N$-methyl-D-aspartate receptormediated modulation of the aortic baroreceptor reflex in the rat nucleus tractus solitarii. Neurosci. Lett. 87, 69-74.

Kubo, T., Amano, M., Ishizuka, T., Ozaki, S., 1993. Beta-alanine and taurine microinjected into the rat caudal ventrolateral medulla increase blood pressure. Clin. Exp. Hypertension 15, 585-597.

Lan, C.T., Wu, W.C., Ling, E.A., Chai, C.Y., 1997. Evidence of a direct projection from the cardiovascular-reactive dorsal medulla to the intermediolateral cell column of the spinal cord in cats as revealed by light and electron microscopy. Neuroscience 77, 521-533.

Li, Y.W., Blessing, W.W., 1990. Localization of vasodepressor neurons in the caudal ventrolateral medulla in the rabbit. Brain Res. 517, 57-63.

Li, Y.W., Gieroba, Z.J., McAllen, R.M., Blessing, W.W., 1991. Neurons in rat caudal ventrolateral medulla inhibit bulbospinal barosensitive neurons in rostral medulla. Am. J. Physiol. 261, R44-R51.

Lin, A.M.Y., Lue, H.M., Lin, R.H., Wang, Y., Pan, C.M., Kuo, J.S., Chai, C.Y., 1988. Paramedian reticular nucleus-sympathetic inhibition in spontaneously hypertensive rats. Brain Res. Bull. 21, 651-657.

Lin, A.M.Y., Wang, Y., Kuo, J.S., Chai, C.Y., 1989. Homocysteic acid elicits pressor responses from ventrolateral medulla and dorsomedial medulla. Brain Res. Bull. 22, 627-631.

Macrae, I.M., Reid, J.L., 1988. Cardiovascular significance of neuropeptide $\mathrm{Y}$ in the caudal ventrolateral medulla of the rat. Brain Res. 456, $1-8$.

Masuda, N., Terui, N., Koshiya, N., Kumada, M., 1991. Neurons in the caudal ventrolateral medulla mediate the arterial baroreceptor reflex by inhibiting barosensitive reticulospinal neurons in the rostral ventrolateral medulla in rabbits. J. Auton. Nerv. Syst. 34, 103-118.

Olney, J.W., 1978. Neurotoxicity of Excitatory Amino Acids. In: McGeer, E.G., Olney, J.W., McGeer, P.L. (Eds.), Kainic acid as a tool in neurobiology, Raven Press, New York, pp. 95-121.

Possas, O.S., Campos, R.R. Jr., Cravo, S.L., Lopes, O.U., Guertzenstein, P.G., 1994. A fall in arterial blood pressure produced by inhibition of the caudalmost ventrolateral medulla: The caudal pressor area. J. Auton. Nerv. Syst. 49, 235-245.

Sesoko, S., Muratani, H., Takishita, S., Teruya, H., Kawazoe, N., Fukiyama, K., 1995. Modulation of baroreflex function by angiotensin II endogenous to the caudal ventrolateral medulla. Brain Res. 671, 38-44. 
Su, C.K., Lin, A.M.Y., Lin, R.H., Kuo, J.S., Chai, C.Y., 1989. Contribution between dorsal and ventrolateral regions of medulla oblongata in vasomotor function of cats. Brain Res. Bull. 23, 447-456.

Terui, N., Masuda, N., Saeki, Y., Kumada, M., 1990. Activity of barosensitive neurons in the caudal ventrolateral medulla that send axonal projections to the rostral ventrolateral medulla in rabbits. Neurosci. Lett. 118, 211-214.

Wang, S.D., Yen, K.T., Pan, C.M., Liu, J.C., Kuo, J.S., Chai, C.Y., 1996. Descending pressor pathways from the dorsomedial and ventrolateral medulla of cats. Chin. J. Physiol. 39, 31-42.

Willette, R.N., Barcas, P.P., Krieger, A.J., Sapru, H.N., 1983. Vasopressor and depressor areas in the rat medulla. Neuropharmacology 22, 1071-1079.

Willette, R.N., Punnen, S., Krieger, A.J., Sapru, H.N., 1984a. Interdependence of rostral and caudal ventrolateral medullary areas in the control of blood pressure. Brain Res. 321, 169-174.

Willette, R.N., Barcas, P.P., Krieger, A.J., Sapru, H.N., 1984b. Endogenous GABAergic mechanisms in the medulla and the regulation of blood pressure. J. Pharmacol. Exp. Ther. 230, 34-39.

Willette, R.N., Punnen-Grandy, S., Krieger, A.J., Sapru, H.N., 1987. Differential regulation of regional vascular resistance by the rostral and caudal ventrolateral medulla in the rat. J. Auton. Nerv. Syst. 18, $143-151$.

Wu, W.C., Tsai, M.L., Liu, C.P., Chai, C.Y., 1995. A device supplementing narishige PE-2M glass microelectrode puller for making better multibarrel micropipettes. Biomed. Engineering-Applications, Basis and Comm. 7, 519-523.

Yang, J.M., Ho, C.W., Lin, R.H., Lin, R.T., Lin, M.T., Yin, T.H., Chai, C.Y., 1992. Reduction of blood $\mathrm{PO}_{2}$ decrease and $\mathrm{PCO}_{2}$ increase during asphyxia by paramedian reticular nucleus in cats. Brain Res. Bull. 29, 573-579.

Yardley, C.P., Andrade, J.M., Weaver, L.C., 1989. Evaluation of cardiovascular control by neurons in the dorsal medulla of rats. J. Auton. Nerv. Syst. 29, 1-12.

Yen, C.T., Hwang, J.C., Su, C.K., Lin, Y.F., Yang, J.M., Chai, C.Y., 1991. Differential actions of the medial region of caudal medulla on autonomic nerve activities. Clin. Exp. Pharmacol. Physiol. 18, 743751.

Yen, C.T., Yen, K.D., Tung, C.S., Chai, C.Y., 1995. Evoked potential elicited by periaqueductal grey stimulation in the pressor sites of dorsal and ventrolateral medulla in cats. Chin. J. Physiol. 38, 171-176. 